\title{
Changes in myocardial echo amplitude during reversible ischaemia in humans
}

David A Lythall, Derek G Gibson, Sudhir S Kushwaha, Michael S Norell, Andrew G Mitchell, Charles J D Ilsley

\begin{abstract}
Objective-This study investigated the changes in regional myocardial ultrasonic backscatter, measured as myocardial echo amplitude, that occur during reversible myocardial ischaemia in humans.
\end{abstract}

Design-Left anterior descending coronary angioplasty was used to produce reversible myocardial ischaemia in human subjects. Regional myocardial echo amplitude was studied in the interventricular septum and left ventricular posterior free wall before, during, and after coronary occlusion with the angioplasty balloon. Wall motion analysis of the left ventricle was performed from simultaneous cross sectional echocardiographic imaging. Patients were studied prospectively.

Patients-Six patients (mean age 56 (SD 11), range 46 to 69 years) with single vessel, left anterior descending coronary artery stenoses, were investigated during elective coronary angioplasty. A total of 11 balloon inflations were studied.

Setting-All patient studies were performed at Harefield Hospital. Echo amplitude analysis was performed at the Royal Brompton Hospital.

Interventions-Angioplasty was performed by the usual procedure at Harefield Hospital for elective coronary angioplasty. All routine medication including $\beta$ blockers and calcium antagonists were continued. Inflation pressures were up to $12 \mathrm{~atm}(1212 \mathrm{kPa})$ and mean inflation time ranged from 30 to 120 (86 (31)) s. In four studies the first inflation was examined, in three the second, in two the third, and in one each the fourth and fifth inflations. Echo amplitude and cross sectional echocardiographic studies were recorded with a 3.5 MHz Advanced Technology Laboratories (ATL) (720A/8736 series) mechanical sector scanner and an ATL Mark II (860-1 series) echocardiograph system with 45 dB logarithmic grey scale compression.

Main outcome measures-Regional echo amplitude was examined in four regions of the left ventricle-namely, the basal and mid-septum, and basal and mid-posterior wall. Consecutive end diastolic and end systolic frames were analysed and cyclic variation was determined as the difference between the level of echo amplitude at end diastole and at end systole. Measurements were made before balloon inflation, at peak inflation, and after balloon deflation. Regional wall motion and systolic wall thickening were analysed qualitatively.

Results-Before balloon inflation, cyclic variation in echo amplitude was noted in all regions (basal septum, $2 \cdot 4$ (SD 1.1) dB; mid-septum, 2.5 (1.1) dB; basal posterior wall, $3.3(2 \cdot 1) \mathrm{dB}$; midposterior wall, $3.9(1.6) \mathrm{dB})$. During balloon inflation there was a significant fall in cyclic variation to $0.4(0.9) \mathrm{dB}$ $(p<0.0002)$ in the mid-septum. This was predominantly owing to an increase in end systolic echo amplitude from 5.4 (2.0) dB to $9.3(1.9) \mathrm{dB}(\mathrm{p} \leqslant 0.01)$. This was associated with the development of severe hypokinesis or akinesis in the mid-septum. No significant changes in echo amplitude occurred in the three other regions examined. Changes were completely reversed after balloon deflation.

Conclusions-These results suggest a causal relation between occlusion of the supplying coronary artery and blunting of myocardial echo amplitude cyclic variation. It is suggested that balloon occlusion produced myocardial ischaemia. The resultant impairment of myocardial contraction then caused a blunting of cyclic variation in echo amplitude. The results of this study provide further data about the ability of quantitative studies of ultrasonic backscatter to identify alterations in the myocardium during injury.

A link between cyclic variation in echo amplitude and cardiac contraction has been shown. ${ }^{1}$ To assess this relation further a human model of reversible myocardial ischaemia was used to examine the effect of a reversible perturbation of cardiac contraction on cyclic variation of echo amplitude. Percutaneous transluminal coronary angioplasty of the left anterior descending artery provided an ideal model for studying left ventricular performance and function during short, controlled, and reversible episodes of myocardial ischaemia. Catheter balloon inflation performed during coronary angioplasty produces virtually total interruption of regional coronary blood flow. Numerous studies during coronary angioplasty have 
Table 1 Coronary angioplasty patient characteristics

\begin{tabular}{llll}
\hline Patient & Sex & Age & $\begin{array}{l}\text { Total number of } \\
\text { inflations studied }\end{array}$ \\
\hline 1 & M & 46 & 1 \\
2 & M & 47 & 1 \\
3 & F & 69 & 2 \\
4 & M & 50 & 2 \\
5 & F & 54 & 3 \\
6 & M & 69 & 2 \\
\hline
\end{tabular}

shown the effect of balloon occlusion on global and regional left ventricular function using either $M$ mode $^{2}$ or cross sectional echocardiography, ${ }^{3-5}$ direct cine left ventriculography, ${ }^{6-8}$ and intravenous digital subtraction ventriculography. ${ }^{9}$ Similarly left ventricular filling dynamics have been studied during coronary angioplasty with high fidelity manometer tipped catheters ${ }^{8}$ and Doppler echocardiography. ${ }^{10}$ These studies suggested that coronary angioplasty provided an ideal model in which to study the effect of reversible myocardial ischaemia on cyclic variation in echo amplitude. As left anterior descending coronary artery angioplasty had been shown to produce changes in segmental shortening in the distal and mid-intraventricular septum, ${ }^{5}$ cyclic variation in echo amplitude was examined in this region of the myocardium in a series of patients with single vessel left anterior descending stenoses who were undergoing coronary angioplasty.

\section{Patients and methods \\ PATIENTS}

Six patients with single vessel left anterior descending coronary artery disease, defined as greater than $70 \%$ luminal stenosis, were investigated during elective coronary angioplasty. No patient had a history of transmural myocardial infarction. Two patients were women and patient age ranged from 46 to 69 years (mean (SD) 56 (11) years). All patients previously had diagnostic coronary angiography and cine left ventriculography in the $30^{\circ}$ right anterior oblique projection performed for the investigation of angina. In all cases left ventriculography was normal or showed minimal anterior hypokinesia only. A total of 11 balloon inflations were studied in the six patients; three inflations in one, two inflations in three, and one inflation each in the remaining two. The study was approved by the hospital ethical committee and all patients gave informed consent. Care

Table 2 Characteristics of angioplasty balloon inflation, electrocardiographic changes, and pain

\begin{tabular}{llcll}
\hline Patient No & $\begin{array}{l}\text { Inflation } \\
\text { studied }\end{array}$ & $\begin{array}{l}\text { Duration of } \\
\text { inflation }(s)\end{array}$ & $\begin{array}{l}\text { ST segment } \\
\text { change }\end{array}$ & $\begin{array}{l}\text { Cardiac } \\
\text { pain }\end{array}$ \\
\hline 1 & 1 & 90 & No & No \\
2 & 1 & 40 & Yes & Yes \\
3 & 1 & 30 & Yes & Yes \\
3 & 2 & 90 & Yes & No \\
4 & 2 & 60 & Yes & No \\
4 & 4 & 120 & No & No \\
5 & 1 & 90 & No & No \\
5 & 3 & 100 & No & No \\
5 & 5 & 120 & No & No \\
6 & 2 & 90 & & \\
6 & 3 & 120 & &
\end{tabular}

was taken not to interfere with or prolong the therapeutic procedure. Table 1 shows characteristics of the patients.

\section{PERCUTANEOUS TRANSLUMINAL CORONARY}

ANGIOPLASTY PROTOCOL

Angioplasty was performed by the usual procedure for elective coronary angioplasty at Harefield Hospital. Patients were premedicated with intramuscular papavertetum and metoclopramide. All routine medication including $\beta$ blockers and calcium antagonists was continued.

The angioplasty procedure was performed via the femoral approach. An intravenous single dose of 5000 units of heparin was administered at the start of the procedure. Non-ionic contrast medium, steerable guide wires, and balloon catheters with diameters varying from 2.5 to $3.5 \mathrm{~mm}$ were used. Inflation pressures were up to 12 atmospheres (1212 $\mathrm{kPa}$ ) and mean inflation time (86 (SD 31)) ranged from 30 to $120 \mathrm{~s}$. Table 2 shows that in four studies the first inflation was examined, in three the second, in two the third, and in one each the fourth and fifth inflations.

Continuous electrocardiographic monitoring was performed throughout the procedure with the six limb and augmented limb leads (I, II, III, aVL, aVF, and aVR) being monitored by a 7 series Seimens Elema Mingograf. The electrocardiogram was recorded immediately before and throughout each balloon inflation and after balloon deflation until any changes had resolved.

ECHO AMPLITUDE AND CROSS SECTIONAL

ECHOCARDIOGRAPHY STUDIES

Echo amplitude and cross sectional echocardiographic studies were recorded with a 3.5 MHz Advanced Technology Laboratories (ATL) (720A/8736 series) mechanical sector scanner and an ATL Mark III (860-1 series) echocardiograph system with $45 \mathrm{~dB}$ logarithmic grey scale compression. To obtain the least modified echo amplitude signal, self correcting circuits were inactivated to avoid further differentiation of the original echo signal. Images were simultaneously displayed in grey scale and in colour by a Brompton Encoder (Alltek Hospital Supplies, Shepperton, Middlesex, UK) as they were recorded. On the colour scale each level of echo amplitude was represented as a single colour in the sequence: cyan, green, yellow, red, magenta, blue, and white, the last corresponding to maximum amplitude. The brightness of the displayed signal was modulated so that within each colour level, the luminosity was varied. This approach allowed a continuous range of echo amplitude to be displayed, and also meant that regions of low echo amplitude appeared with low brightness on the screen. These were less conspicuous on the final image and thus preserved the original amplitude perspective. ${ }^{11}$

All images were acquired with a standardised protocol to enable intrapatient and interpatient comparisons. Patients were studied in the supine position and long axis parasternal views were recorded. Gain settings were standard- 
ised: the master gain was increased until the parietal pericardium posterior to the left ventricle was just displayed at the highest level of the encoder (white). The depth compensation was then set to a linear ramp across the image at a rate of roughly $2 \mathrm{~dB} / \mathrm{cm}$. The gain settings were not altered thereafter. Images were stored on $U$-matic $3 / 4$ inch video tape in a Sony VO 5800PS recorder.

Image analysis was performed by a Tandon PCA desk top computer. Stop frame end diastolic and end systolic images for analysis were selected by a video recorder with digital frame advance (Sony VO 5800PS). These frames were transferred from tape to a $512 \times 256 \times 6$ bit frame store. Images were processed with an adaptive filtering process for the reduction of speckle as this decreased echo amplitude variance and allowed smaller sample sizes to be used..$^{12}{ }^{13}$ Four areas of interest were defined, in the interventricular septum (basal and mid-septum), and posterior wall (basal and mid-posterior wall) as defined by the left ventricular anatomical nomenclature of Edwards et $a l^{14}$ and measurements of 256 individual picture elements (pixels) were made from each area. Four separate measurements, each of 64 pixels, were made and averaged rather than one individual measurement of 256 pixels as this allowed the pixel matrix to be placed within the mid-myocardium and thus avoided including specular echoes from the endocardium or epicardium. A maximum pixel intensity for each image was derived by measuring the maximum colour intensity of the superimposed colour scale (usually corresponding to 52 levels of grey scale). This corresponded to a value of $45 \mathrm{~dB}$ and allowed echo amplitude values (dB) to be assigned (a grey scale level thus corresponding to approximately $0.86 \mathrm{~dB}$ ). Individual pixel amplitudes were displayed as a histogram and a separate digital readout displayed mean, median, and SD values for each region measured. The display of the frequency histogram on the image proved useful, as an obviously bimodal form usually meant that specular echoes had mistakenly been included in the area of interest. Consecutive end diastolic and end systolic frames were analysed and cyclic variation determined as the

Table 3 Mean end diastolic and end systolic echo amplitude values during percutaneous transluminary coronary angioplasty (mean (SD))

\begin{tabular}{clll}
\hline & $\begin{array}{l}\text { Pre- } \\
\text { inflation }\end{array}$ & $\begin{array}{l}\text { Peak } \\
\text { inflation }\end{array}$ & $\begin{array}{l}\text { Post- } \\
\text { inflation }\end{array}$ \\
\hline Basal septum: & & & \\
ED (dB) & $7.5(1.9)$ & $7.8(1.4)$ & $7.0(1.3)$ \\
ES (dB) & $5.1(1.2)$ & $5.9(1.6)$ & $4.6(0.8)$ \\
Mid-septum: & & & \\
ED (dB) & $8.4(2.2)$ & $9.7(2.1)$ & $7.9(2 \cdot 1)$ \\
ES (dB) & $5.9(2.0)$ & $9.3(1.9)^{\star}$ & $5.4(1.2)$ \\
Basal PW: & & & \\
ED (dB) & $8.1(2.4)$ & $7.9(1.7)$ & $7.2(1.7)$ \\
ES (dB) & $4.7(1.0)$ & $5.0(0.8)$ & $4.2(0.9)$ \\
Mid-PW: & & & \\
ED (dB) & $9 \cdot 1(2.0)$ & $8.5(2.3)$ & $8.1(2.0)$ \\
ES (dB) & $5.2(0.9)$ & $5.6(1.5)$ & $4.6(1.2)$ \\
\hline
\end{tabular}

${ }^{\star} p \leqslant 0.0001$ (ANOVA) for all four groups, $p<0.005$ for all paired groups (Bonferroni's) comparing the mid-septum to other regions. Basal PW, basal posterior wall; ED, end diastole; ES, end systole; Mid-PW, mid-posterior wall. difference between the level of echo amplitude at end diastole and at end systole. Cyclic variation index was calculated as the ratio: cyclic variation (end diastolic echo amplitude minus end systolic echo amplitude): end diastolic echo amplitude.

Images for analysis were recorded at baseline with the deflated balloon and guide wire across the coronary lesion, at the peak of balloon inflation and after balloon deflation. In order not to interfere with the therapeutic procedure and patient safety and welfare, the timing of the imaging after deflation was by necessity variable. Seven balloon inflation images were acquired 15-30 seconds after deflation, but in the remaining four inflations, images could not be acquired until five, 10, 20, and 60 minutes after balloon deflation. In one study images of the mid-posterior wall were not suitable for analysis.

Regional wall motion analysis was performed qualitatively. Normal left ventricular wall motion was assessed by the amount of systolic myocardial thickening and inward endocardial motion. Abnormalities of segmental wall function were identified by a reduction in wall thickening and motion.

\section{Results}

CLINICAL OUTCOME, CHEST PAIN, AND ST SEGMENT CHANGES

All six patients underwent successful dilatation of their coronary stenoses. Significant ST segment shifts on the monitored electrocardiographic leads occurred during five balloon inflations. This was accompanied by chest pain consistent with cardiac ischaemia during three inflations (table 2). On all occasions ST segment changes and chest pain resolved rapidly after balloon deflation.

\section{WALL MOTION AND THICKENING \\ ABNORMALITIES}

Before balloon inflation no significant wall motion abnormalities were present. During each of the 11 balloon inflations, severe hypokinesis or akinesis developed in the midseptum and distal septum accompanied by diminished or absent wall thickening. Obvious hyperkinesis or severe hypokinesis of the ventricular wall was not seen elsewhere in the myocardium during any balloon inflation, except in the distal mid-posterior wall of two patients (patients 1 and 2). In the seven inflations where imaging was obtained for the first 30 seconds after balloon deflation the hypokinesis or akinesis seen resolved within the $30 \mathrm{~s}$ after deflation. For the remaining four inflations, resolution of the wall motion abnormalities was noted at the time of imaging after deflation.

\section{END DIASTOLIC AND END SYSTOLIC REGIONAL} ECHO AMPLITUDE

Table 3 shows mean values for regional echo amplitude for all four regions of ventricular myocardium at both end diastole and end systole. Tables 4-7 show the individual values for all patients. Values were greatest at end 
Table 4 Individual echo amplitude $(d B)$ at the basal septum for end diastole (ED) and end systole (ES) during angioplasty

\begin{tabular}{|c|c|c|c|c|c|}
\hline Patient & Variable & $\begin{array}{l}\text { Inflation } \\
\text { studied } n\end{array}$ & $\begin{array}{l}\text { Pre- } \\
\text { inflation }\end{array}$ & $\begin{array}{l}\text { Peak } \\
\text { inflation }\end{array}$ & $\begin{array}{l}\text { Post- } \\
\text { inflation }\end{array}$ \\
\hline 1 & $\begin{array}{l}\text { ED } \\
\text { ES } \\
\text { CVI }\end{array}$ & 1 & $\begin{array}{l}6 \cdot 2 \\
3 \cdot 4 \\
0 \cdot 45\end{array}$ & $\begin{array}{l}8 \cdot 8 \\
7 \cdot 1 \\
0 \cdot 20\end{array}$ & $\begin{array}{l}6 \cdot 3 \\
4 \cdot 7 \\
0 \cdot 25\end{array}$ \\
\hline 2 & $\begin{array}{l}\text { ED } \\
\text { ES } \\
\text { CVI }\end{array}$ & 1 & $\begin{array}{l}5 \cdot 8 \\
4 \cdot 6 \\
0 \cdot 20\end{array}$ & $\begin{array}{l}5 \cdot 9 \\
4 \cdot 0 \\
0 \cdot 32\end{array}$ & $\begin{array}{l}6 \cdot 8 \\
4 \cdot 4 \\
0 \cdot 35\end{array}$ \\
\hline 3 & $\begin{array}{l}\text { ED } \\
\text { ES } \\
\text { CVI }\end{array}$ & 1 & $\begin{array}{l}9 \cdot 3 \\
6 \cdot 1 \\
0 \cdot 35\end{array}$ & $\begin{array}{c}10 \cdot 5 \\
8 \cdot 7 \\
0 \cdot 17\end{array}$ & $\begin{array}{l}6 \cdot 3 \\
5 \cdot 2 \\
0 \cdot 17\end{array}$ \\
\hline 3 & $\begin{array}{l}\text { ED } \\
\text { ES } \\
\text { CVI }\end{array}$ & 2 & $\begin{array}{l}8 \cdot 4 \\
5 \cdot 4 \\
0 \cdot 36\end{array}$ & $\begin{array}{l}7 \cdot 6 \\
5 \cdot 3 \\
0 \cdot 30\end{array}$ & $\begin{array}{l}8 \cdot 7 \\
4 \cdot 4 \\
0 \cdot 50\end{array}$ \\
\hline 4 & $\begin{array}{l}\text { ED } \\
\text { ES } \\
\text { CVI }\end{array}$ & 2 & $\begin{array}{l}5 \cdot 2 \\
3 \cdot 2 \\
0 \cdot 37\end{array}$ & $\begin{array}{l}6 \cdot 1 \\
5 \cdot 4 \\
0 \cdot 11\end{array}$ & $\begin{array}{l}5 \cdot 9 \\
3.6 \\
0.39\end{array}$ \\
\hline 4 & $\begin{array}{l}\text { ED } \\
\text { ES } \\
\text { CVI }\end{array}$ & 4 & $\begin{array}{l}5 \cdot 4 \\
4 \cdot 1 \\
0 \cdot 24\end{array}$ & $\begin{array}{l}8.0 \\
7.6 \\
0.05\end{array}$ & $\begin{array}{l}4 \cdot 8 \\
3 \cdot 8 \\
0 \cdot 20\end{array}$ \\
\hline 5 & $\begin{array}{l}\text { ED } \\
\text { ES } \\
\text { CVI }\end{array}$ & 1 & $\begin{array}{c}11.6 \\
7.0 \\
0.39\end{array}$ & $\begin{array}{l}9 \cdot 0 \\
6 \cdot 4 \\
0 \cdot 29\end{array}$ & $\begin{array}{l}7.9 \\
3.8 \\
0.52\end{array}$ \\
\hline 5 & $\begin{array}{l}\text { ED } \\
\text { ES } \\
\text { CVI }\end{array}$ & 3 & $\begin{array}{l}8 \cdot 6 \\
5 \cdot 8 \\
0 \cdot 32\end{array}$ & $\begin{array}{l}8 \cdot 1 \\
5 \cdot 7 \\
0 \cdot 30\end{array}$ & $\begin{array}{l}9 \cdot 0 \\
6 \cdot 2 \\
0 \cdot 31\end{array}$ \\
\hline & $\begin{array}{l}\text { ED } \\
\text { ES } \\
\text { CVI }\end{array}$ & 5 & $\begin{array}{l}7 \cdot 9 \\
6 \cdot 4 \\
0 \cdot 20\end{array}$ & $\begin{array}{l}7 \cdot 7 \\
6.5 \\
0 \cdot 15\end{array}$ & $\begin{array}{l}7 \cdot 4 \\
5 \cdot 3 \\
0 \cdot 28\end{array}$ \\
\hline 6 & $\begin{array}{l}\text { ED } \\
\text { ES } \\
\text { CVI }\end{array}$ & 2 & $\begin{array}{l}6 \cdot 7 \\
5 \cdot 3 \\
0 \cdot 21\end{array}$ & $\begin{array}{l}6 \cdot 0 \\
4 \cdot 3 \\
0 \cdot 29\end{array}$ & $\begin{array}{l}7 \cdot 3 \\
4 \cdot 8 \\
0 \cdot 34\end{array}$ \\
\hline 6 & $\begin{array}{l}\text { ED } \\
\text { ES } \\
\text { CVI }\end{array}$ & 3 & $\begin{array}{l}7 \cdot 6 \\
4 \cdot 6 \\
0 \cdot 40\end{array}$ & $\begin{array}{l}7 \cdot 7 \\
3 \cdot 4 \\
0.55\end{array}$ & $\begin{array}{l}6 \cdot 7 \\
4 \cdot 3 \\
0 \cdot 35\end{array}$ \\
\hline
\end{tabular}

CVI, cyclic variation index. inflation $(\mathrm{p}<0.01)$ After balloon deflation, values fell significantly $(p<0.01)$ and returned to values not significantly different from preinflation values.

\section{CYCLIC VARIATION IN ECHO AMPLITUDE}

Table 8 shows mean values for cyclic variation. Tables 4-7 shows individual values for cyclic variation index. Values for pre-inflation and post-inflation were not significantly different for the four regions for either the absolute value of cyclic variation of echo amplitude (end diastolic echo amplitude minus end systolic echo amplitude) or the cyclic variation index. At peak inflation there was a significant fall in both these variables in the region of the midseptum (ANOVA, $p \leqslant 0.0001$ ) compared with both pre-inflation (Bonferroni's (comparison of pairs), $p \leqslant 0.0002$ and $p \leqslant 0.01$ respectively) and post inflation (Bonferroni's, $p \leqslant 0.0002$ and $\mathrm{p} \leqslant 0.01$ ) values. This major decrease in cyclic variation was not seen in the other three regions of myocardium examined (figure).

\section{SUMMARY OF RESULTS}

During balloon inflation the mid-septum and distal septum of the ventricle became severely hypokinetic or akinetic. These changes were accompanied by significant changes in echo amplitude in the mid-septum. In this region a noticeable increase in end systolic echo amplitude occurred resulting in a fall in cyclic variation in echo amplitude to $16 \%$ of preinflation values and a similar fall in cyclic variation index to $10 \%$ of pre-inflation values. These changes paralleled the decrease in wall thickening seen qualitatively. After balloon deflation these changes reverted to pre-inflation values as did wall thickening. systolic values of echo amplitude were greatly increased by peak inflation compared with pre-

Table 5 Individual echo amplitude ( $d B$ ) at the mid-septum for end diastole (ED) and end systole (ES) during angioplasty

\begin{tabular}{|c|c|c|c|c|c|}
\hline Patient & Variable & $\begin{array}{l}\text { Inflation } \\
\text { studied } n\end{array}$ & $\begin{array}{l}\text { Pre- } \\
\text { inflation }\end{array}$ & $\begin{array}{l}\text { Peak } \\
\text { inflation }\end{array}$ & $\begin{array}{l}\text { Post- } \\
\text { inflation }\end{array}$ \\
\hline 1 & $\begin{array}{l}\text { ED } \\
\text { ES } \\
\text { CVI }\end{array}$ & 1 & $\begin{array}{l}7 \cdot 8 \\
4 \cdot 0 \\
0 \cdot 49\end{array}$ & $\begin{array}{l}9 \cdot 8 \\
9 \cdot 4 \\
0 \cdot 04\end{array}$ & $\begin{array}{l}6 \cdot 5 \\
5 \cdot 7 \\
0 \cdot 11\end{array}$ \\
\hline 2 & $\begin{array}{l}\text { ED } \\
\text { ES } \\
\text { CVI }\end{array}$ & 1 & $\begin{array}{l}7 \cdot 2 \\
5 \cdot 6 \\
0 \cdot 22\end{array}$ & $\begin{array}{c}8.8 \\
9.0 \\
-0.03\end{array}$ & $\begin{array}{l}5 \cdot 9 \\
3 \cdot 7 \\
0 \cdot 37\end{array}$ \\
\hline 3 & $\begin{array}{l}\text { ED } \\
\text { ES } \\
\text { CVI }\end{array}$ & 1 & $\begin{array}{c}11.8 \\
8.6 \\
0.27\end{array}$ & $\begin{array}{c}10.9 \\
8.7 \\
0.20\end{array}$ & $\begin{array}{c}10 \cdot 2 \\
6 \cdot 1 \\
0 \cdot 41\end{array}$ \\
\hline 3 & $\begin{array}{l}\text { ED } \\
\text { ES } \\
\text { CVI }\end{array}$ & 2 & $\begin{array}{l}9 \cdot 5 \\
5 \cdot 7 \\
0.39\end{array}$ & $\begin{array}{c}11.4 \\
11.0 \\
0.04\end{array}$ & $\begin{array}{l}9 \cdot 8 \\
5 \cdot 9 \\
0 \cdot 40\end{array}$ \\
\hline 4 & $\begin{array}{l}\text { ED } \\
\text { ES } \\
\text { CVI }\end{array}$ & 2 & $\begin{array}{l}5 \cdot 0 \\
3 \cdot 3 \\
0.33\end{array}$ & $\begin{array}{c}5.6 \\
6.1 \\
-0.09\end{array}$ & $\begin{array}{l}5 \cdot 3 \\
3 \cdot 4 \\
0 \cdot 35\end{array}$ \\
\hline 4 & $\begin{array}{l}\text { ED } \\
\text { ES } \\
\text { CVI }\end{array}$ & 4 & $\begin{array}{l}5 \cdot 3 \\
4 \cdot 1 \\
0 \cdot 23\end{array}$ & $\begin{array}{c}7 \cdot 2 \\
7 \cdot 4 \\
-0.03\end{array}$ & $\begin{array}{l}5 \cdot 1 \\
4 \cdot 0 \\
0 \cdot 21\end{array}$ \\
\hline 5 & $\begin{array}{l}\text { ED } \\
\text { ES } \\
\text { CVI }\end{array}$ & 1 & $\begin{array}{l}9.5 \\
6.9 \\
0.27\end{array}$ & $\begin{array}{c}11.3 \\
11.4 \\
-0.02\end{array}$ & $\begin{array}{l}9 \cdot 0 \\
6 \cdot 2 \\
0 \cdot 31\end{array}$ \\
\hline 5 & $\begin{array}{l}\text { ED } \\
\text { ES } \\
\text { CVI }\end{array}$ & 3 & $\begin{array}{l}9 \cdot 6 \\
7 \cdot 7 \\
0 \cdot 20\end{array}$ & $\begin{array}{c}9.8 \\
10.5 \\
-0.07\end{array}$ & $\begin{array}{c}10.6 \\
6.9 \\
0.35\end{array}$ \\
\hline 5 & $\begin{array}{l}\text { ED } \\
\text { ES } \\
\text { CVI }\end{array}$ & 5 & $\begin{array}{c}11 \cdot 2 \\
9 \cdot 4 \\
0.16\end{array}$ & $\begin{array}{c}13.0 \\
12.4 \\
0.05\end{array}$ & $\begin{array}{l}9 \cdot 9 \\
6 \cdot 6 \\
0 \cdot 34\end{array}$ \\
\hline 6 & $\begin{array}{l}\text { ED } \\
\text { ES } \\
\text { CVI }\end{array}$ & 2 & $\begin{array}{l}7 \cdot 2 \\
5 \cdot 5 \\
0 \cdot 24\end{array}$ & $\begin{array}{c}10.0 \\
9.4 \\
0.07\end{array}$ & $\begin{array}{l}7 \cdot 0 \\
5 \cdot 7 \\
0 \cdot 19\end{array}$ \\
\hline 6 & $\begin{array}{l}\text { ED } \\
\text { ES } \\
\text { CVI }\end{array}$ & 3 & $\begin{array}{l}9 \cdot 0 \\
4 \cdot 3 \\
0.52\end{array}$ & $\begin{array}{l}8.9 \\
7.5 \\
0 \cdot 16\end{array}$ & $\begin{array}{l}8 \cdot 0 \\
5 \cdot 2 \\
0 \cdot 35\end{array}$ \\
\hline
\end{tabular}

CVI, cyclic variation index.

\section{Discussion}

This study documents the effects of reversible coronary artery occlusion on cyclic variation of myocardial echo amplitude. In the mid-septal region of the left ventricular myocardium, after balloon occlusion of the supplying left anterior descending coronary artery, regional cyclic variation in echo amplitude was much blunted, falling to values less than $16 \%$ of pre-occlusion values. Cyclic variation index, which relates the level of cyclic variation to end diastolic levels of echo amplitude, also declined significantly to less than $10 \%$ of pre-occlusion values. These changes were completely reversible after balloon deflation suggesting a causal relation between occlusion of the supplying vessel and the blunting of cyclic variation. It is postulated that balloon coronary artery occlusion produced myocardial ischaemia in the territory of the left anterior descending coronary artery. The resultant impairment of myocardial contraction subsequently caused a blunting of cardiac cycle dependent variation in backscattered ultrasound. After balloon deflation and reperfusion these changes were rapidly reversed with no permanent sequelae. The evidence for this postulate is discussed further. 
Table 6 Individual echo amplitude $(d B)$ at the basal posterior wall for end diastole (ED) and end systole (ES) during angioplasty

\begin{tabular}{|c|c|c|c|c|c|}
\hline Patient & Variable & $\begin{array}{l}\text { Inflation } \\
\text { studied } n\end{array}$ & $\begin{array}{l}\text { Pre- } \\
\text { inflation }\end{array}$ & $\begin{array}{l}\text { Peak } \\
\text { inflation }\end{array}$ & $\begin{array}{l}\text { Post- } \\
\text { inflation }\end{array}$ \\
\hline 1 & $\begin{array}{l}\text { ED } \\
\text { ES } \\
\text { CVI }\end{array}$ & 1 & $\begin{array}{l}7 \cdot 2 \\
4 \cdot 2 \\
0 \cdot 42\end{array}$ & $\begin{array}{l}7 \cdot 6 \\
6 \cdot 5 \\
0 \cdot 15\end{array}$ & $\begin{array}{l}6 \cdot 7 \\
4 \cdot 6 \\
0 \cdot 32\end{array}$ \\
\hline 2 & $\begin{array}{l}\text { ED } \\
\text { ES } \\
\text { CVI }\end{array}$ & 1 & $\begin{array}{l}7 \cdot 2 \\
4 \cdot 6 \\
0 \cdot 37\end{array}$ & $\begin{array}{l}8 \cdot 5 \\
4 \cdot 4 \\
0 \cdot 48\end{array}$ & $\begin{array}{l}6 \cdot 0 \\
4 \cdot 1 \\
0 \cdot 31\end{array}$ \\
\hline 3 & $\begin{array}{l}\text { ED } \\
\text { ES } \\
\text { CVI }\end{array}$ & 1 & $\begin{array}{l}6 \cdot 6 \\
5 \cdot 1 \\
0 \cdot 22\end{array}$ & $\begin{array}{l}6.8 \\
4.7 \\
0.31\end{array}$ & $\begin{array}{l}7.9 \\
3.6 \\
0.54\end{array}$ \\
\hline 3 & $\begin{array}{l}\text { ED } \\
\text { ES } \\
\text { CVI }\end{array}$ & 2 & $\begin{array}{l}6.9 \\
4 \cdot 2 \\
0.40\end{array}$ & $\begin{array}{l}7.0 \\
4.5 \\
0.36\end{array}$ & $\begin{array}{l}6.9 \\
3.6 \\
0.48\end{array}$ \\
\hline 4 & $\begin{array}{l}\text { ED } \\
\text { ES } \\
\text { CVI }\end{array}$ & 2 & $\begin{array}{l}5.0 \\
3.6 \\
0.28\end{array}$ & $\begin{array}{l}5.7 \\
3.9 \\
0.31\end{array}$ & $\begin{array}{l}4 \cdot 7 \\
3 \cdot 2 \\
0 \cdot 31\end{array}$ \\
\hline 4 & $\begin{array}{l}\text { ED } \\
\text { ES } \\
\text { CVI }\end{array}$ & 4 & $\begin{array}{l}5.8 \\
3.9 \\
0.33\end{array}$ & $\begin{array}{l}5 \cdot 8 \\
4 \cdot 0 \\
0 \cdot 31\end{array}$ & $\begin{array}{l}4.5 \\
3.3 \\
0.25\end{array}$ \\
\hline 5 & $\begin{array}{l}\text { ED } \\
\text { ES } \\
\text { CVI }\end{array}$ & 1 & $\begin{array}{c}11 \cdot 7 \\
7 \cdot 1 \\
0.40\end{array}$ & $\begin{array}{c}11.4 \\
5.0 \\
0.56\end{array}$ & $\begin{array}{l}9.0 \\
4.6 \\
0.50\end{array}$ \\
\hline 5 & $\begin{array}{l}\text { ED } \\
\text { ES } \\
\text { CVI }\end{array}$ & 3 & $\begin{array}{c}10.7 \\
5 \cdot 1 \\
0.52\end{array}$ & $\begin{array}{l}9 \cdot 1 \\
5 \cdot 4 \\
0 \cdot 40\end{array}$ & $\begin{array}{l}9 \cdot 3 \\
4 \cdot 3 \\
0 \cdot 54\end{array}$ \\
\hline 5 & $\begin{array}{l}\text { ED } \\
\text { ES } \\
\text { CVI }\end{array}$ & 5 & $\begin{array}{c}11.8 \\
4.0 \\
0.66\end{array}$ & $\begin{array}{c}10.0 \\
5.7 \\
0.44\end{array}$ & $\begin{array}{l}8 \cdot 8 \\
6.0 \\
0 \cdot 32\end{array}$ \\
\hline 6 & $\begin{array}{l}\text { ED } \\
\text { ES } \\
\text { CVI }\end{array}$ & 2 & $\begin{array}{l}6 \cdot 9 \\
5 \cdot 7 \\
0 \cdot 17\end{array}$ & $\begin{array}{l}7 \cdot 1 \\
5 \cdot 7 \\
0 \cdot 20\end{array}$ & $\begin{array}{l}7 \cdot 4 \\
5 \cdot 6 \\
0 \cdot 25\end{array}$ \\
\hline 6 & $\begin{array}{l}\text { ED } \\
\text { ES } \\
\text { CVI }\end{array}$ & 3 & $\begin{array}{l}8.4 \\
4.1 \\
0.52\end{array}$ & $\begin{array}{l}8 \cdot 2 \\
5 \cdot 2 \\
0 \cdot 37\end{array}$ & $\begin{array}{l}8 \cdot 7 \\
3.6 \\
0.59\end{array}$ \\
\hline
\end{tabular}

CVI, cyclic variation index

\section{EVIDENCE FOR MYOCARDIAL ISCHAEMIA \\ Clinical findings}

An early manifestation of acute myocardial ischaemia is the development of ST segment change on the electrocardiogram. Such changes were noted during five of the 11 balloon occlusions in this study. Previous studies in which limb lead electrocardiograms only were monitored have shown similar findings. Hauser et al found ST segment shift in seven of 16 patients undergoing left anterior descending

Table 7 Individual echo amplitude (dB) at the mid-posterior wall for end diastole (ED) and end systole (ES) during angioplasty

\begin{tabular}{|c|c|c|c|c|c|}
\hline Patients & Variable & $\begin{array}{l}\text { Inflation } \\
\text { studied } n\end{array}$ & $\begin{array}{l}\text { Pre- } \\
\text { inflation }\end{array}$ & $\begin{array}{l}\text { Peak } \\
\text { inflation }\end{array}$ & $\begin{array}{l}\text { Post- } \\
\text { inflation }\end{array}$ \\
\hline 1 & $\begin{array}{l}\text { ED } \\
\text { ES } \\
\text { CVI }\end{array}$ & 1 & $\begin{array}{l}7 \cdot 5 \\
4 \cdot 2 \\
0 \cdot 44\end{array}$ & $\begin{array}{c}6.0 \\
6.2 \\
-0.04\end{array}$ & $\begin{array}{l}6 \cdot 2 \\
4 \cdot 0 \\
0 \cdot 35\end{array}$ \\
\hline 2 & $\begin{array}{l}\text { ED } \\
\text { ES } \\
\text { CVI }\end{array}$ & 1 & $\begin{array}{l}8 \cdot 7 \\
6 \cdot 0 \\
0 \cdot 33\end{array}$ & $\begin{array}{c}8.8 \\
9.2 \\
-0.04\end{array}$ & $\begin{array}{l}8 \cdot 5 \\
7 \cdot 1 \\
0 \cdot 16\end{array}$ \\
\hline 3 & $\begin{array}{l}\text { ED } \\
\text { ES } \\
\text { CVI }\end{array}$ & 1 & $\begin{array}{l}8.9 \\
5.5 \\
0.38\end{array}$ & $\begin{array}{l}7.6 \\
4.9 \\
0.36\end{array}$ & $\begin{array}{l}8 \cdot 8 \\
5 \cdot 8 \\
0 \cdot 34\end{array}$ \\
\hline 3 & $\begin{array}{l}\text { ED } \\
\text { ES } \\
\text { CVI }\end{array}$ & 2 & $\begin{array}{l}8 \cdot 2 \\
4 \cdot 6 \\
0 \cdot 45\end{array}$ & $\begin{array}{l}7.0 \\
4.5 \\
0.37\end{array}$ & $\begin{array}{l}7 \cdot 5 \\
4 \cdot 4 \\
0 \cdot 42\end{array}$ \\
\hline 4 & $\begin{array}{l}\text { ED } \\
\text { ES } \\
\text { CVI }\end{array}$ & 2 & $\begin{array}{l}\star \\
\star \\
\star\end{array}$ & $\begin{array}{l}\star \\
\star \\
\star\end{array}$ & $\begin{array}{l}\star \\
\star \\
\star\end{array}$ \\
\hline 4 & $\begin{array}{l}\text { ED } \\
\text { ES } \\
\text { CVI }\end{array}$ & 4 & $\begin{array}{l}6 \cdot 2 \\
4 \cdot 7 \\
0 \cdot 25\end{array}$ & $\begin{array}{l}5.6 \\
4.5 \\
0.20\end{array}$ & $\begin{array}{l}4 \cdot 1 \\
4 \cdot 4 \\
0 \cdot 12\end{array}$ \\
\hline 5 & $\begin{array}{l}\text { ED } \\
\text { ES } \\
\text { CVI }\end{array}$ & 1 & $\begin{array}{c}12 \cdot 3 \\
6.9 \\
0.44\end{array}$ & $\begin{array}{c}12 \cdot 5 \\
6 \cdot 2 \\
0 \cdot 50\end{array}$ & $\begin{array}{c}11.5 \\
4.8 \\
0.58\end{array}$ \\
\hline 5 & $\begin{array}{l}\text { ED } \\
\text { ES } \\
\text { CVI }\end{array}$ & 3 & $\begin{array}{c}11.2 \\
5.7 \\
0.50\end{array}$ & $\begin{array}{l}9 \cdot 8 \\
6 \cdot 4 \\
0 \cdot 35\end{array}$ & $\begin{array}{l}9.0 \\
3.6 \\
0.60\end{array}$ \\
\hline 5 & $\begin{array}{l}\text { ED } \\
\text { ES } \\
\text { CVI }\end{array}$ & 5 & $\begin{array}{c}10 \cdot 3 \\
5 \cdot 0 \\
0.52\end{array}$ & $\begin{array}{l}9.9 \\
5.9 \\
0.41\end{array}$ & $\begin{array}{l}8 \cdot 6 \\
3.9 \\
0.55\end{array}$ \\
\hline 6 & $\begin{array}{l}\text { ED } \\
\text { ES } \\
\text { CVI }\end{array}$ & 2 & $\begin{array}{l}7 \cdot 4 \\
5 \cdot 2 \\
0 \cdot 30\end{array}$ & $\begin{array}{l}6 \cdot 7 \\
5 \cdot 2 \\
0 \cdot 23\end{array}$ & $\begin{array}{l}7 \cdot 6 \\
5 \cdot 4 \\
0 \cdot 28\end{array}$ \\
\hline 6 & $\begin{array}{l}\text { ED } \\
\text { ES } \\
\text { CVI }\end{array}$ & 3 & $\begin{array}{c}10 \cdot 4 \\
4 \cdot 1 \\
0.61\end{array}$ & $\begin{array}{c}10 \cdot 7 \\
3 \cdot 7 \\
0 \cdot 66\end{array}$ & $\begin{array}{l}9 \cdot 3 \\
3 \cdot 8 \\
0 \cdot 59\end{array}$ \\
\hline
\end{tabular}

^Not analysable; CVI, cyclic variation index. coronary angioplasty. ${ }^{3}$ Visser et al found ST segment change in four of 15 patients undergoing coronary angioplasty ( 10 single vessel left anterior descending, four single vessel right coronary artery, and one double vessel right coronary artery and left anterior descending coronary artery). ${ }^{4}$ The use of 12 lead electrocardiographic monitoring greatly increases the frequency of ST segment change noted. In a group of 32 patients with mixed single and multiple vessel disease of whom 17 had angioplasty of the left anterior descending coronary artery, Labovitz et al analysed electrocardiograms to find significant ST segment changes in 25 of $31 . .^{10}$ Wohlgelernter et al detected ST segment changes in 12 of 14 patients during angioplasty of the left anterior descending coronary artery and Norell $e t a l$ in 33 of 52 patients. ${ }^{9}$ The use of intracoronary electrograms, recorded via the tip of the angioplasty guide wire positioned distal to the coronary stenosis being treated, greatly increased the yield of ST segment changes. ${ }^{15}$ i6 In a study by Jain and Gettes ST segment change occurred in 33 of 35 patients undergoing left anterior descending coronary angioplasty but was simultaneously detected in only 13 patients by observation of surface leads II and $\mathrm{V}_{5}{ }^{16} \mathrm{~T}$ The absence of ST segment change in over half (six of 11) of the balloon occlusions examined in our study does not therefore exclude acute myocardial ischaemia during those occlusions. Similarly the occurrence of chest pain during only three of 11 balloon inflations was consistent with that previously reported. Hauser et $a l^{\beta}$ reported chest pain in eight of 16 patients during left anterior descending coronary angioplasty, and Visser $e t$ $a l^{4}$ in five of 15 patients although only 11 of these patients underwent left anterior descending coronary angioplasty, the remainder being right coronary artery lesions. The absence of chest pain would appear to correlate poorly with the absence of acute myocardial ischaemia, chest pain being the last manifestation of ischaemia. ${ }^{3}$

\section{Wall motion and systolic wall thickening}

Coronary artery ligation in open chested dogs causes echocardiographically detectable systolic wall thinning in the ischaemic region within 30 s. ${ }^{17}{ }^{18}$ In our study, decreased systolic wall thickening was noted in the distal and midinterventricular septum during all 11 balloon inflations studied. Similar findings have been well documented by other workers. Hauser $e t$ al found new or increased wall motion abnormalities in 14 of 16 patients during left anterior descending coronary angioplasty. ${ }^{3}$ Hypokinesia was noted within $33(19(7)) \mathrm{s}$ of balloon occlusion in all 14 patients and invariably preceded ST segment shift or chest pain where these occurred. Wall motion returned to normal within 30 seconds of balloon deflation in 12 of these patients. Alam et al studied eight patients with left anterior descending single vessel coronary artery disease during 14 balloon occlusions and noted a fall in systolic wall thickening of $50 \%$ or more in the interventricular septum during 13 of these occlusions. ${ }^{2}$ 
Table 8 Mean (SD) cyclic variation in echo amplitude and cyclic variation index during angioplasty

\begin{tabular}{|c|c|c|c|c|}
\hline & $\begin{array}{l}\text { Pre-inflation } \\
(d B)\end{array}$ & $\begin{array}{l}\text { Peak inflation } \\
(d B)\end{array}$ & $\begin{array}{l}\text { Post inflation } \\
(d B)\end{array}$ & $\begin{array}{l}\text { p Value } \\
\text { for all paired } \\
\text { groups (Boniferoni) }\end{array}$ \\
\hline \multicolumn{5}{|c|}{ Cyclic variation in echo ampitude } \\
\hline $\begin{array}{l}\text { Basal septum } \\
\text { Mid-septum } \\
\text { Basal PW } \\
\text { Mid-PW }\end{array}$ & $\begin{array}{l}2.4(1 \cdot 1) \\
2.5(1 \cdot 1) \\
3.3(2 \cdot 1) \\
3.9(1 \cdot 6)\end{array}$ & $\begin{array}{l}1.9(1.0) \\
0.4(0.9)^{\star} \\
2.9(1.6) \\
2.8(2.5)\end{array}$ & $\begin{array}{l}2.4(1 \cdot 1) \\
2.5(1.2) \\
3.0(1.5) \\
3.5(2.0)\end{array}$ & $\begin{array}{l}p<0.005 \\
p<0.005 \\
p<0.005 \\
p<0.005\end{array}$ \\
\hline \multicolumn{5}{|c|}{ Cyclic variation index } \\
\hline $\begin{array}{l}\text { Basal septum } \\
\text { Mid-septum } \\
\text { Basal PW } \\
\text { Mid-PW }\end{array}$ & $\begin{array}{l}0.32(0.09) \\
0.30(0.12) \\
0.39(0.14) \\
0.42(0.11)\end{array}$ & $\begin{array}{l}0.25(0.14) \\
0.03(0.09)^{\star} \\
0.35(0.12) \\
0.30(0.21)\end{array}$ & $\begin{array}{l}0.33(0.11) \\
0.31(0.10) \\
0.40(0.13) \\
0.40(0.18)\end{array}$ & $\begin{array}{l}\mathrm{p}<0.01 \\
\mathrm{p}<0.01 \\
\mathrm{p}<0.01 \\
\mathrm{p}<0.01\end{array}$ \\
\hline
\end{tabular}

${ }^{\star} p \leqslant 0.0001$ (ANOVA) for all four groups, comparing the mid-septum with other regions. Basal $P W$, basal posterior wall, Mid-PW, mid-posterior wall.

Wall thickening abnormalities began within $20 \mathrm{~s}$ of balloon inflation in all 13 cases and returned to pre-occlusion levels within $20 \mathrm{~s}$ of balloon deflation. Visser et al documented similar wall motion abnormalities developing in 15 patients during 49 balloon inflations at a mean of 8 (3) $\mathrm{s}$ after balloon inflation. ${ }^{4}$ Wall motion returned to baseline in all patients 19 (8) s after balloon deflation. Again Wohlgelernter et al reported 14 patients each undergoing angioplasty of an isolated left anterior descending stenosis who all developed severe hypokinesia $(29 \%)$ or akinesia $(71 \%)$ of the septal and apical region of the left ventricle by balloon inflation lasting $60 \mathrm{s.}{ }^{5}$ Recovery to pre-occlusion values began within 15 (5) $\mathrm{s}$ (range 6 to 22) and was complete in all patients by $70 \mathrm{~s}$ after inflation with a mean time to full recovery of 43 (17) s. Evidence that ischaemia is the cause of the wall motion abnormalities recorded echocardiographically was provided by techniques to improve myocardial perfusion distal to the occluding angioplasty balloon. Timmis et al infused arterial blood through the angioplasty catheter into the distal coronary artery in 10 patients during $60 \mathrm{~s}$ balloon occlusions. ${ }^{19}$ These inflations were compared

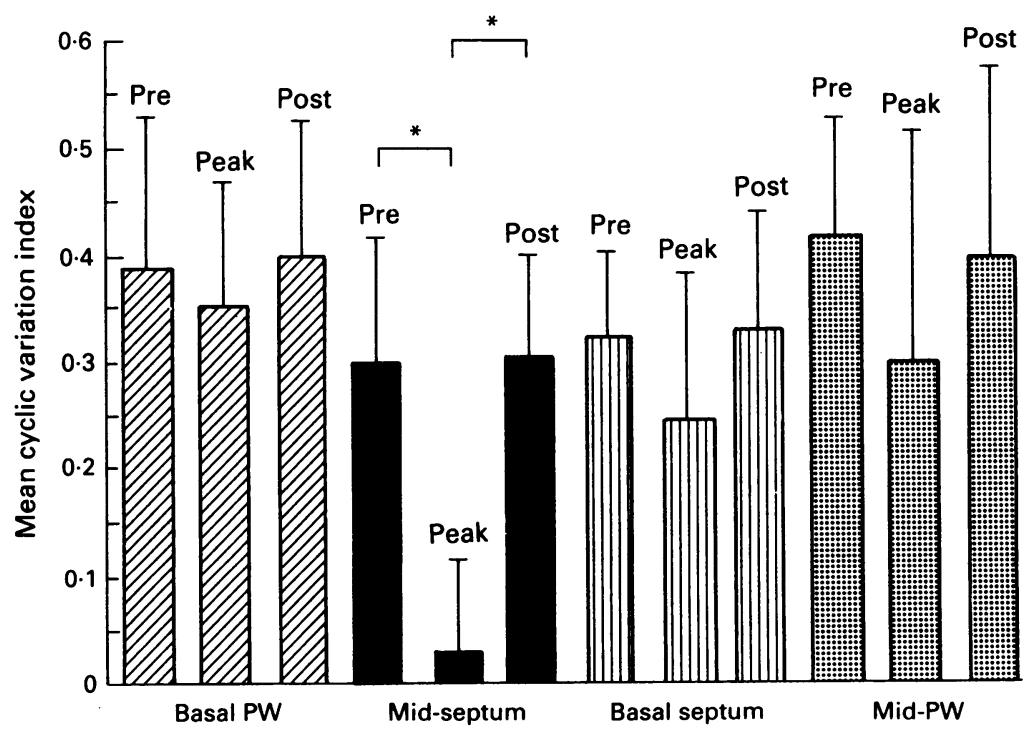

Mean $(S D)$ cyclic variation index during coronary angioplasty. During balloon inflation in the left anterior descending artery there was a significant fall in echo amplitude cyclic variation index in the mid-septal region only. After balloon deflation and the return of systolic myocardial thickening, cyclic variation index was restored to pre-inflation values. ${ }^{\star} p, \leqslant 0.01 ; P W$, posterior wall. with control occlusions with either no distal infusion or distal infusion with Hartman's solution. All dilatations without blood infusion in the 10 patients produced regional wall motion abnormalities within 16.0 (3.2) s. By contrast only one dilatation with distal blood infusion produced wall motion abnormalities. Infusion with Hartman's was not helpful in limiting wall motion abnormalities suggesting that oxygen delivery via arterial blood, rather than wash out of metabolites, was responsible for the benefit. Jaffe et al studied 42 patients undergoing coronary angioplasty using distal transcatheter coronary perfusion with oxygenated Fluosol DA ( $20 \%$ emulsion), a perfluorocarbon oxygen transport fluid. ${ }^{20}$ They found a dramatic fall in left ventricular regional wall motion abnormality during perfusion with oxygenated Fluosol DA compared with controls. It seems that the development of regional wall motion abnormalities during coronary angioplasty not only reflects myocardial ischaemia but is also a sensitive and early marker of it. The development of regional wall motion abnormalities during coronary angioplasty in this study seem to reflect underlying myocardial ischaemia in the territory supplied by the left anterior descending coronary artery.

\section{CYCLIC VARIATION IN REGIONAL}

BACKSCATTERED ULTRASOUND

In 1983 and 1984, Madaras et al and Barzilai et al first reported cyclic variation in backscattered myocardial ultrasound. ${ }^{21} 22$ They analysed myocardial integrated backscatter as a measure of backscattered ultrasound and examined this in a dog model after occlusion of the left anterior descending artery. Cyclic variation in integrated backscatter was significantly blunted in ischaemic areas of myocardium. These areas were previously delineated at open thoracotomy during brief coronary occlusion. The effects of reperfusion were not studied in this model. In a later study using a similar open chest dog model, cyclic variation in integrated backscatter was examined $10 \mathrm{~min}$ after coronary occlusion and after two hours of reperfusion. Cyclic variation at the left ventricular apex in the four dogs studied was $5.6(1.4) \mathrm{dB}$ at baseline and fell to $0.4(1.5) \mathrm{dB}$ after $10 \mathrm{~min}$ of occlusion $(\mathrm{p}<0.02)$. After two hours of reperfusion, values were not significantly different from baseline at 3.9 (1.2) dB. Cross sectional echocardiographic apical four chamber views of the apex showed dyskinesis and loss of systolic thickening after $10 \mathrm{~min}$ of occlusion. This was substantially but not completely restored to baseline values after two hours of reperfusion. Corresponding values for systolic wall thickening at the apex were; baseline, $63.4(17 \cdot 7) \%$; 10 min occlusion, $2 \cdot 2$ $(11.5) \%(\mathrm{p}<0.001)$; two hours reperfusion, $52.9(26.8) \%$ (NS). Similar effects of ischaemia on cyclic variation of backscattered ultrasound from dog myocardium after coronary artery occlusion have been found by several other workers. $^{23-25}$

Cyclic variation in backscattered myocardial ultrasound has also been found in humans. 
This has been shown by integrated backscatter, ${ }^{26-29}$ grey scale echo amplitude, ${ }^{30}$ and colour encoded echo amplitude. ${ }^{1}$ The effect of ischaemia on cyclic variation in backscattered ultrasound in humans is limited. Milunski et al studied 21 patients in a cardiac care unit within the first 24 hours after the onset of symptoms suggestive of acute myocardial infarction. ${ }^{28}$ Areas of ischaemic injury were identified by cross sectional echocardiography and electrocardiography. Cyclic variation of integrated backscatter was measured in these areas and normal control areas of myocardium. Thrombolytic therapy was given to 17 patients. Cyclic variation in normal regions was $4.8(0.5)$ dB compared with $0.8(0.3) \mathrm{dB}(\mathrm{p}<0.05)$ in regions with infarction. In the 15 patients who were serially studied and also underwent coronary angiography to define vessel patency, 10 had patent infarct related coronary arteries. In these patients the magnitude of cyclic variation increased with time $(1.3(0.6) \mathrm{dB}$ to 2.5 $(0.5) \mathrm{dB}, \mathrm{p}<0.05)$ where in those with occluded infarct related vessels no significant recovery was seen $(0.3(0.3) \mathrm{dB}$ to $0.6(0.3) \mathrm{dB}$, NS). It was concluded that the restoration of cyclic variation in the patent vessel group represented the beneficial effect of reperfusion. Significantly the improvement in cyclic variation shown in this group was not accompanied by a noticeable improvement in wall motion.

It is probably incorrect, however, to compare the brief periods of coronary occlusion during clinical angioplasty with the prolonged periods of coronary occlusion in the study of Milunski et $a l,{ }^{28}$ and the animal studies already mentioned in which coronary occlusion times greater than five min were used. Many investigators have shown that although partial recovery of myocardial contractile function after brief ischaemia occurs promptly, complete recovery may require longer periods of reperfusion. ${ }^{31-33}$ With more prolonged periods of myocardial ischaemia ( $>5 \mathrm{~min}$ ) it is likely that structural changes are occurring. Myofibril stretching after similar periods of ischaemia are well described ${ }^{34}$ as are other ultrastructural changes. ${ }^{35}$ Zhao et al found disruption of the collagen matrix of dog myocardium with accompanying persistent myocardial dysfunction, after 12 sequential five min occlusions of the left anterior descending coronary artery..$^{36}$ This was unaccompanied by irreversible cellular damage. Therefore it is not surprising that cyclic variation may not be fully restored after several hours of reperfusion if ischaemia is prolonged more than a few minutes with resultant damage to cellular and extracellular structures. This contrasts with the rapid recovery of both myocardial systolic function and cyclic variation in echo amplitude that occurred with coronary reperfusion, after balloon deflation during angioplasty, which this study documents.

One other study, by Hajduczki et $a l^{\beta 7}$ has documented changes in backscattered ultrasound during coronary angioplasty in humans. They examined patients during left anterior descending coronary angioplasty and showed a substantial change in cyclic variation of back- scatter during balloon inflation. Backscatter fell from $8.6(3.3)$ units pre-inflation to $3.2(1.7)$ units at peak inflation $(p<0.001)$. They did not comment on whether this reversed after balloon deflation. They also noted an increase in cyclic variation of backscatter in areas of myocardium that were identified as hyperkinetic during balloon inflation. In our study the grouped data did not show a significant increase in cyclic variation of backscattered ultrasound in either basal segments of the left ventricle (where hyperkinesis would be most likely to occur) during balloon inflation, although increases in cyclic variation index of $>10 \%$ were noted in both areas during two of the 11 inflations (tables 4,6 ).

There are several explanations for the changes in cyclic variation that occur in association with myocardial ischaemia. Wickline et al have suggested a mechanism to explain cyclic variation in backscattered myocardial ultrasound (in their case integrated backscatter). ${ }^{38}$ They suggested a relation between cyclic variation in integrated backscatter and myocardial contractile function which reflects cyclic alterations in myofibrillar elastic parameters, with the juxtaposition of intracellular and extracellular elastic elements that have different intrinsic acoustic impedances providing an appropriately sized scattering interface at the cellular level. Because acoustic impedance mismatch is partially determined by elastic modulus, changes in local elastic moduli which result from myocardial elastic elements not obeying Hook's law as they are stretched, may alter the degree of impedance mismatch. With a simple Maxwell type muscle model to depict cardiac cell mechanical behaviour, a model employing an extracellular parallel elastic element and intracellular series elastic and contractile elements can be constructed. This model predicts the decrease in backscattered ultrasound found during systole. Cardiac cycle dependent alterations in the degree of local acoustic impedance mismatch may therefore elicit concomitant changes in backscattered ultrasound. More recently Rijsterborgh et al confirmed in animal studies that the higher systolic backscatter seen during ischaemia could be explained by the decrease in wall thickness that occurs with ischaemia and not by the ischaemia itself, in keeping with the influence of myocardial contraction on cyclic variation. $^{39}$ Other possibilities include alterations in the relative insonifying angle due to changing fibre angle and shape during the cardiac cycle as it is known that both reflection and attenuation of ultrasound alter according to the direction of fibre insonification. Whatever the exact basis of cyclic variation in backscattered ultrasound, there is no doubt an important relation with contractile events occuring within the myocardium at either a cellular or fibre level. As myocardial ischaemia occurs these contractile events are disrupted and cyclic variation as a result is blunted. Changes in myocardial blood flow during coronary occlusion must also be considered as a cause of the blunting of cyclic variation in 
backscattered ultrasound, as changes in red blood cell concentration have been shown to noticeably affect overall levels (as distinct from cyclic variation) of backscattered ultrasound in experimental models. ${ }^{41-43}$ Wickline et al, however, in a series of experiments on perfused and non-perfused dog hearts that were cyclically distended and relaxed by an intracavitary ventricular balloon, showed that myocardial perfusion with whole blood did not contribute significantly to the phenomenon of cyclic variation in backscattered ultrasound. ${ }^{38}$ Importantly, in this study there was an increase in end diastolic echo amplitude in the midseptum during balloon inflation from $8 \cdot 4(2.2)$ $\mathrm{dB}$ to $9.7(2.1) \mathrm{dB}$ that is significant $(\mathrm{p}<0.05)$ if the mid-septum alone is considered. This would be consistent with a fall in concentration of formed blood elements in the myocardium during coronary occlusion. ${ }^{43}$

\section{Conclusion}

It is likely that balloon coronary occlusion during angioplasty results in reversible myocardial ischaemia which produces a reversible blunting of cardiac cycle dependent backscattered ultrasound after temporary contractile dysfunction induced by ischaemia. The results of this study are consistent with this hypothesis and provide further data on the ability of techniques involved in the quantitative study of backscattered ultrasound to clarify alterations in the myocardium during injury.

DAL was supported as a junior research fellow by the British Heart Foundation, London, United Kingdom.

1 Lythall DA, Gibson DG, Ilsley CD, Yacoub MH. Changes in myocardial echo amplitude related to the cardiac cycle. Eur Heart $J$ 1989;10(suppl):220.

2 Alam M, Khaja F, Brymer J, Marzelli M, Goldstein S. Echocardiographic evaluation of left ventricular function during coronary artery angioplasty. Am J Cardiol 1986 57:20-5.

3 Hauser AM, Gangadharan V, Ramos RG, Gordon S, Timmis GC, Dudlets P. Sequence of mechanical, electrocardiographic and clinical effects of repeated
coronary artery occlusion in human beings: echocoronary artery occlusion in human beings: echocardiographic observations durin
$J$ Am Coll Cardiol 1985;5:193-7.

4 Visser CA, David GK, Kan G, et al. Two-dimensional echocardiography during percutaneous transluminal coronary angioplasty. Am Heart J 1986;111:1035-41.

5 Wohlgelernter D, Cleman M, Highman HA, et al. Regional myocardial dysfunction during coronary angioplasty: evaluation by 2 -dimensional echocardiography and 12 lead elect

6 Doorey AJ, Mehmel HC, Schwartz FX, Kubler W. Amelioration by nitroglycerine of left ventricular ischaemia induced by percutaneous transluminal coronary ischaemia induced by percutaneous transluminal coronary angioplasty: assessment by haemodynamic variables and

7 left ventriculography. $J$ Am Coll Cardiol 1985;6:267-74. Serruys $P W$, Wijns W, Van Den Brand $M$, et al. Left
ventricular performance, regional blood flow, wall motion ventricular performance, regional blood flow, wall motion
and lactate metabolism during transluminal angioplasty. and lactate metabolism during

8 Piscione F, Hugenholtz PG, Serruys PW. Impaired left ventricular filling dynamics during percutaneous transluminal angioplasty for coronary artery disease. $\mathrm{Am} J$ Cardiol 1987;59:29-37.

9 Norell MS, Lyons JP, Gershlick AH, et al. Assessment of left ventricular performance during percutaneous transluminal coronary angioplasty: a study by intravenous digital subt

10 Labovitz AJ, Lewen MC, Kern M, Vandormael M, Deligonal U, Kennedy HL. Evaluation of left ventricular systolic and diastolic dysfunction during transient Cardiol 1987;10:748-55.
11 Logan-Sinclair RB, Wong CM, Gibson DG. Clinical application of amplitude processing of echocardiographic images. Br Heart J 1981;45:621-7.

12 Bamber JC, Daft C. Adaptive filtering for reduction of speckle in ultrasonic pulse-echo images. Ultrasonics 1986; (Jan):41-4.

13 Massey RJ, Logan-Sinclair RB, Bamber JC, Gibson DG. Quantitative effects of speckle reduction on cross sectional echocardiographic images. Br Heart J 1989;62:298-304.

14 Edwards WD, Tajik AJ, Seward JB. Standardized nomenclature and anatomic basis for regional tomographic analysis of the heart. Mayo Clin Proc 1981;56:479-97.

15 Freidman PL, Shook TL, Kirschenbaum JM, Selwyn AP, Ganz $P$. Value of the intracoronary electrogram to monitor myocardial ischaemia during percutaneous transluminal coronary angioplasty. Circulation 1986;74:330-9.

16 Jain A, Gettes LS. Patterns of ST-segment change during acute no-flow myocardial ischaemia produced by balloon occlusion during angioplasty of the left anterior descending coronary artery. Am J Cardiol 1991;67:305-7.

17 Pandian NG, Kieso RA, Kerber RE. Two-dimensional echocardiography in experimental coronary stenosis. II. Relationship between systolic wall thinning and regional myocardial perfusion in severe coronary stenosis. Circulation 1982;66:603-11.

18 Pandian NG, Kerber RE. Two-dimensional echocardiography in experimental coronary stenosis. I. Sensitivity and specificity in detecting transient myocardial and specificity in detecting transient myocardial dyskinesis: comparison

19 Timmis AD, Crick JCP, Griffin B, Sowton E. Prevention of regional myocardial ischaemia during PTCA - the effects of infusing arterial blood. Br Heart J 1989;61:628 (abstr).

20 Jaffe CC, Wohlgelernter D, Cabin H, et al. Preservation of left ventricular ejection fraction during percutaneous transluminal coronary angioplasty by distal transcatheter perfusion of oxygenated Fluosol DA $20 \%$. Am Heart $J$ 1988;115:1156-64

21 Madaras EI, Barzilai B, Perez JE, Sobel BE, Miller JG. Changes in myocardial backscatter throughout the cardiac cycle. Ultrasonic Imaging 1983;5:229-39.

22 Barzilai B, Madaras EI, Sobel BE, Miller JG, Perez JE. Effects of myocardial contraction on ultrasonic backscatter

before and after ischemia. Am J Physiol 1984;247:478-83. JE. Sensitive detection of the effects of reperfusion on JE. Sensitive detection of the effects of reperfusion on
myocardium by ultrasonic tissue characterization with myocardium by ultrasonic tissue characterization

24 Sagar KB, Rhyne TL, Warltier DC, Pelc L, Wann LS. Intramyocardial variability in integrated backscatter: effects of coronary occlusion and reperfusion. Circulation 1987;75:436-42.

25 Milunski MR, Mohr GA, Wear KA, Sobel BE, Miller JG, Wickline SA. Early identification with ultrasonic integrated backscatter of viable but stunned myocardium in dogs. J Am Coll Cardiol 1989;14:462-71.

26 Vered Z, Barzilai B, Mohr GA, et al. Quantitative ultrasonic tissue characterization with real-time integrated backscatter imaging in normal human subjects and in patients scatter imaging in normal human subjects and in patients
with dilated cardiomyopathy. Circulation 1987;5:1067-73

27 Milunski MR, Canter CE, Wickline SA, Sobel BE, Miller JG, Perez JE. Cardiac cycle dependent variation of integrated backscatter is not distorted by abnormal myo-
cardial wall motion in human subjects with paradoxical cardial wall motion in human subjects with paradoxion.
septal motion. Ultrasound Med Biol 1989;15:311-7.

28 Milunski MR, Mohr GA, Perez JE, et al. Ultrasonic tissue Milunski MR, Mohr GA, Perez JE, et al. Ultrasonic tissue
characterization with integrated backscatter: Acute myocharacterization with integrated backscatter: Acute myo-
cardial ischaemia, reperfusion, and stunned myocardium cardial ischaemia, reperfusion, and stunned

29 Masuyama T, Nellessen U, Schnittger I, Tye TL, Haskell WL, Popp RL. Ultrasonic tissue characterization with a real time integrated backscatter imaging system in normal and aging human hearts. J Am Coll Cardiol 1989;14: 1702-8.

30 Olshansky B, Collins SM, Skorton DJ, Prasad NV. Variation of left ventricular myocardial gray level on twodimensional echocardiograms as a result of cardiac contraction. Circulation 1984;6:972-7.

31 Heyndrickx GR, Millar RW, McRitchie R, Maroko PR, Vatner $S$. Regional myocardial function and electrophysiological alterations after brief coronary artery occlusion in conscious dogs. J Clin Invest 1975;56:978-85.

32 Kloner RA, DeBoer LWV, Darsee J, Ingwall JS, Braunwald $D$. Recovery from prolonged abnormalities, of canine myocardium salvaged from ischaemic necrosis by myocardium salvaged from ischaemic necrosis by
coronary reperfusion. Proc Natl Acad Sci USA 1981; coronary rep

33 Weiner JM, Apstein CS, Arthur JH, Pirzada FA, Hood WB Persistence of myocardial injury following brief periods of coronary occlusion. Cardiovasc Res 1976;10:678-86.

34 Jennings RB, Ganote CE. Structural changes in myocardium during acute ischaemia. Circ Res 1974;34/35 (suppl III):156-72.

35 Kloner RA, Ganote CE, Whalen DA, Jennings RB. Effect of a transient period of ischemia on myocardial cells. II. Fine structure during the first few minutes of reflow. $A m$ Pathol 1974;74:399-422.

36 Zhao M, Zhang H, Robinson TF, Factor SM, Sonnenblick EH, Eng C. Profound structural alterations of the extracellular collagen matrix in postischemic dysfunctional ("Stunned") but viable myocardium. J Am Coll Cardiol 1987;10:1322-34.

37 Hajduczki I, Jaffe M, Areeda J, et al. Ultrasonic backscatter and 2D echocardiographic wall motion during 
38 Wickline SA, Thomas LJ III, Miller JG, Sobel BE, Perez JE. A relationship between ultrasonic integrated backscatter and myocardial contractile function. J Clin Imvest 1985;76:2151-60.

39 Rijsterborgh H, Mastik F, Lancé CT, Sassen LMA Verdouw PD, Roelandt J, ( of myocardial wall thickness and ischaemia to ultrason myocardial integrated backscatter during experimenta

40 Aygen M, Popp RL. Influence of the orientation of myocardial fibres on echocardiographic images. Am J Cardio
1987;60:147-52.

41 Shung KK, Siegelmann RA, Reed JM. Scattering of ultrasound

Reid JM. Ultrasonic scattering from tissues. hung KK, Reid JM. Ultrasonic scattering from tissues. Proceedings of the IGEE

43 Mimbs JW, Bauwens D, Cohen RD, O’Donnell M, Miller JG, Sobel BE. Effects of myocardial ischemia on quantitative ultrasonic backscatter and identification of responsible determinants. Circ Res 1981;49:89-96.

\section{PLANTS IN CARDIOLOGY}

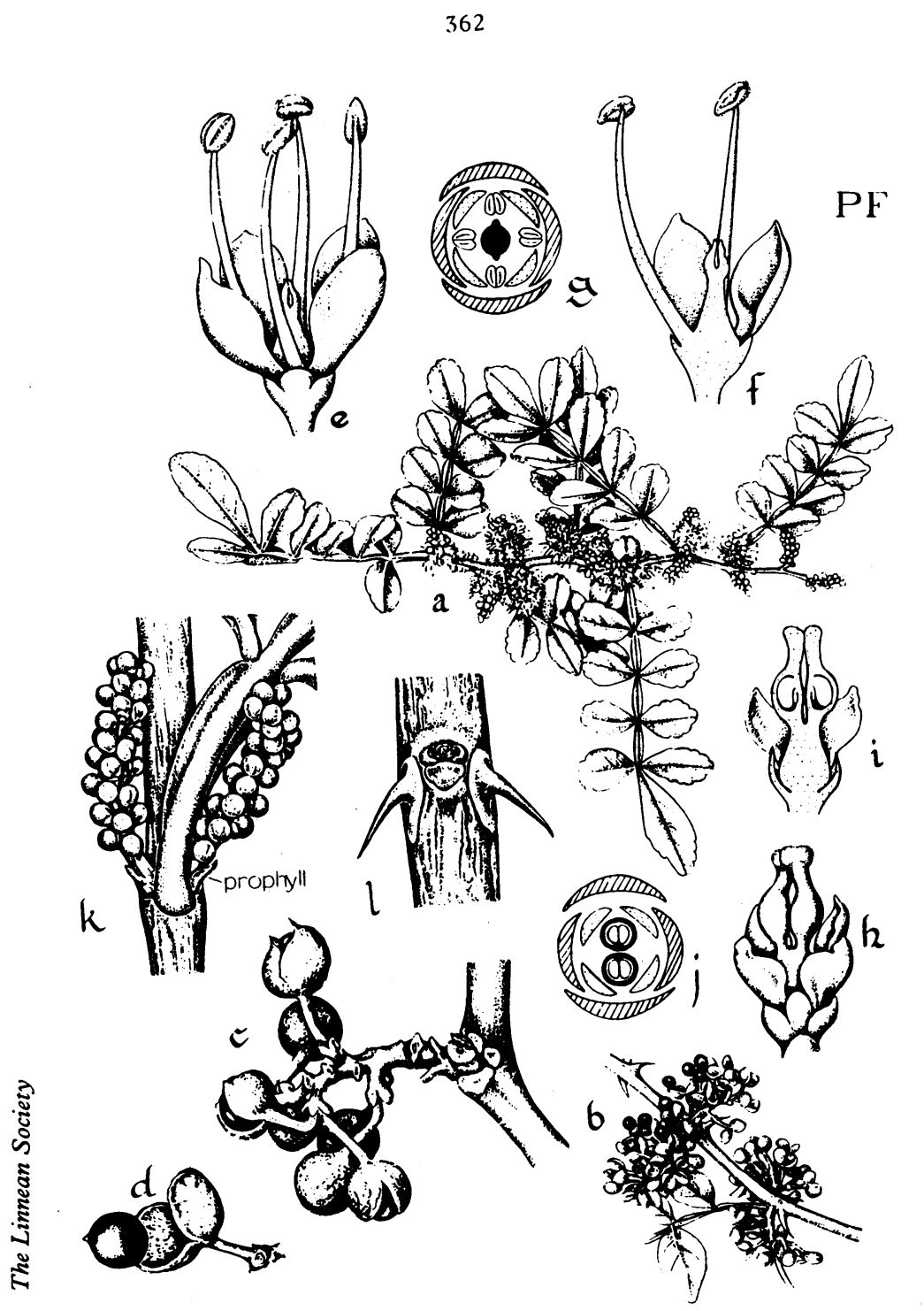

\section{Fagarine}

In 1932 G Stuckert was the first to isolate a new alkaloid, fagarine, from the Argentinian plant Fagara coco (Rutaceae); and with A Sartori he showed that it had a depressant action on the myocardium of rabbits. Further work at the University of Cordoba by Moisset de Espanés and others showed that fagarine raised the threshold for atrial and ventricular fibrillation in response to faradic stimulation, and that it decreased the incidence of ventricular fibrillation after coronary ligation in dogs. In all these experiments it was more effective than quinidine. Then A Taquini tried its effect in six patients with atrial flutter or fibrillation who were resistant to quinidine. In all of them intramuscular fagarine restored sinus rhythm within 30 minutes (Science 1945;102:69-70). In 1948 David Scherf showed that fagarine reliably reverted atrial fibrillation induced by aconitine in dogs (Proceedings of the Society for Experimental Biology and Medicine 1948;67: 59-60).

The genus Fagara has been merged with Zanthoxylum and the name of the original $F$ coco (Gill.) Engl. is now $Z$ coco Gill ex Hook and Arn. The illustration is of a closely related species. The South African plant $Z$ capensis, a "fever tree". is used medicinally.

The family Rutaceae is widespread, especially in the tropics, and it includes Pilocarpus microphyllus, the source of pilocarpine. Citrus fruits belong to this family. Rutaceae is the fourth family of plants described in Plants in Cardiology with antiarrhythmic properties-quinidine, procaine, and lignocaine all being derived from other families. It would be interesting to know whether fagarine is still under investigation. 